\title{
Introduction
}

\section{Le sociologue en globe-trotter}

Réceptions, apports et difficultés de la sociologie de l'international d'Yves Dezalay

\section{Grégory Daho et Antoine Vauchez}

\author{
(2) OpenEdition \\ Journals \\ Édition électronique \\ URL : http://journals.openedition.org/conflits/22058 \\ DOI : $10.4000 /$ conflits. 22058 \\ ISSN : $1777-5345$ \\ Éditeur : \\ CECLS - Centre d'études sur les conflits - Liberté et sécurité, L'Harmattan \\ Édition imprimée \\ Date de publication : 15 décembre 2020 \\ Pagination : 7-16 \\ ISBN : 978-2-343-22588-3 \\ ISSN : $1157-996 \mathrm{X}$ \\ Référence électronique \\ Grégory Daho et Antoine Vauchez, « Le sociologue en globe-trotter », Cultures \& Conflits [En ligne], \\ 119-120 | automne-hiver 2020, mis en ligne le 15 février 2021, consulté le 01 avril 2021. URL: http:// \\ journals.openedition.org/conflits/22058 ; DOI : https://doi.org/10.4000/conflits.22058
}




\title{
Le sociologue en globe-trotter
}

\author{
Réceptions, apports et difficultés \\ de la sociologie de l'international d'Yves Dezalay
}

\section{Grégory DAHO, Antoine VAUCHEZ}

Grégory Daho est maître de conférences en Science Politique de l'Université Paris 1 Panthéon-Sorbonne et membre du Centre européen de sociologie et de science politique (CESSP).

Antoine Vauchez est directeur de recherche au CNRS, membre du Centre européen de sociologie et science politique (CESSP), Université Paris 1 PanthéonSorbonne / EHESS.

\begin{abstract}
u fil de nombreuses enquêtes et de plusieurs centaines d'entretiens

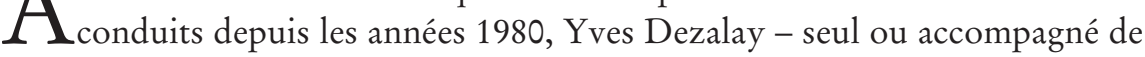
son acolyte Bryant Garth ${ }^{1}-$ a tracé une voie originale au cœur des sciences sociales de l'international. Ancré dans la sociologie des champs et des capitaux, mais maintenant une posture toujours éclectique sur le plan théorique, il a construit une sociologie incarnée de la mondialisation saisie au prisme des stratégies d'internationalisation et de reproduction des élites périphériques et des transformations des savoirs d'État. Évitant de s'enfermer dans la bibliographie et les questionnements d'une seule chapelle (sociologie des élites, relations internationales, sociologie du droit, sociologie de l'État, sociologie des professions, etc.), ses travaux forment aujourd'hui une ouvre aussi singulière qu'incontournable pour qui entend étudier ce que "l'internationalisation » fait aux champs du pouvoir, à leurs élites et aux formes de légitimité qui y ont cours. Pour preuve sans doute de leur fécondité, on les retrouve aujourd'hui
\end{abstract}

1. Il est difficile et probablement vain de chercher à dissocier ici les deux chercheurs du point de vue de leurs contributions respectives, tant leur itinéraire de recherche a été partagé depuis plus de vingt-cinq ans. C'est d'autant plus inutile qu'au fil des enquêtes en commun, la construction en binôme (juriste-sociologue, européen/français-américain, etc.) est devenue partie intégrante de la stratégie de recherche elle-même comme des conditions de publication et de réception : sur ce point, Bigo D., Cohen A., «Enquêter sur l'internationalisation des noblesses d'État. Retour réflexif sur des stratégies de double jeu. Entretien avec Yves Dezalay », Cultures E Conflits, nº9, 2015, pp. 15-52. 
mobilisés par nombre de jeunes chercheurs investis sur des terrains très différents et dans une grande diversité de sous-champs d'enquête (area studies, études postcoloniales, études européennes, travaux sur l'américanisation, politique des droits de l'homme, philanthropie, élites internationales, etc.) ${ }^{2}$.

Paradoxalement pourtant, la portée de ce travail a rarement été discutée en tant que telle ${ }^{3}$. Si quelques publications récentes ont certes permis de retracer plus spécifiquement la trajectoire intellectuelle d'Yves Dezalay ${ }^{4}$, on peine encore à positionner cette contribution dans l'espace désormais foisonnant des sciences sociales de l'international, sans doute du fait de l'éclectisme de l'auteur lui-même, qui puise dans différentes traditions et construit ses objets au croisement de diverses littératures, mais aussi du fait de son peu d'intérêt pour l'exercice devenu aujourd'hui canonique de «l'état de l'art » et du cadrage théorique ${ }^{5}$. Bientôt trente ans après la publication de Marchands de droit (1992) qui marquait une première ouverture de la sociologie des champs aux terrains internationaux ${ }^{6}$, ce dossier souhaite faire le point sur les acquis, la portée, les usages possibles, mais aussi certains angles morts de ses travaux. Construit dans le sillage d'un colloque organisé par le Centre européen de sociologie et de science politique (CESSP) à l'Université Paris 1 PanthéonSorbonne en janvier 2018, il conduit à interroger la fécondité mais aussi les limites de ses hypothèses sur les processus d'internationalisation, la circulation et la reproduction des élites de la mondialisation comme sur les conditions de leur transposition à de nouveaux terrains d'enquête. S'il s'agit bien par ce biais de rendre hommage à un in(c)lassable chercheur sillonnant sans relâche les terrains de l'international (il poursuit aujourd'hui ses travaux du côté des transformations internationales des filières de formation des élites juridiques), le dossier ne tient pas des Mélanges. Il veut en effet tirer parti des travaux de l'auteur pour interroger plus globalement l'état des sciences sociales de l'international, du point de vue des théories sociologiques de la mondialisation, des stratégies d'ouverture de terrains et d'enquête ou de l'ou-

2. On retrouvait du reste cette diversité lors du colloque « Le sociologue en globe-trotter. Réceptions et usages de la sociologie d'Yves Dezalay » organisé à l'Université Paris 1 les 18 et 19 janvier 2018, à l'origine de ce numéro thématique. Nombre des communications provenaient de doctorants et de jeunes docteurs (Paris 1, EHESS, Paris Dauphine, Lille 2), mais aussi de collègues étrangers (Danemark, Canada, Brésil, Argentine, Grande Bretagne).

3. Voir néanmoins, Siméant-Germanos J., "Champs internationaux et transformations du pouvoir d'État : en lisant Dezalay et Garth ", Revue française de science politique, vol. 53, n5, octobre 2003, pp. 819-824, et Munger F., "Globalization through the Lens of Palace Wars: What Elite Lawyers' Careers Can and Cannot Tell Us about Globalization of Law”, Law and social inquiry, vol. 37, $\mathrm{n}^{\circ} 2,2012$, pp. 476-499; et les articles dans ce dossier de FrançoisXavier Dudouet et Antoine Vion, ainsi que de Mikael Madsen.

4. Cf. Boussard V., «Entretien avec Yves Dezalay », Sociologies pratiques, vol. 21, n², 2010, pp. 13-18, et surtout Bigo D., Cohen A., « Enquêter sur l'internationalisation des noblesses d'État », op. cit.

5. Yves Dezalay parle ainsi lui-même "d'une démarche théorique relativement tardive». Voir Dezalay Y., « La sociologie des champs en mode globe-trotter : d'un apprentissage sur le tas à une théorisation sur le tard ", à paraître.

6. Dezalay Y., Marchands de droit, Paris, Fayard, 1992. 
tillage analytique et conceptuel à disposition des chercheurs contemporains.

\section{De la sociologie du droit à la sociologie de la globalisation (et retour)}

C'est en ouvrant la boîte du droit et des professions juridiques qu'Yves Dezalay est entré dans la sociologie de l'international. Tiré de son doctorat, l'ouvrage Marchands de droit marque une première incursion dans l'analyse de champs transnationaux - un terrain que Pierre Bourdieu lui-même n'avait alors que très rarement envisagé et exploré autrement que de façon programmatique ${ }^{7}$. En pointant les alliances passées entre les fractions modernisatrices du droit français et les cabinets juridiques multinationaux anglo-américains, il ouvrait la voie à un nouveau programme de recherche qui retrace les batailles et alliances transnationales, indissociablement sociales, professionnelles et politiques, autour de la définition de la «bonne gouvernance ». Yves Dezalay y fait voir ainsi comment les stratégies d'internationalisation des juristes de droit des affaires issus des law firms anglo-américaines « impactent » dans les années 1980 une Europe continentale dont les champs juridiques européens ont historiquement placé le droit des affaires aux marges - forçant « l'élite des clercs » à un difficile aggiornamento des hiérarchies internes au droit. Son deuxième livre, Dealing in Virtue. International Commercial Arbitration and the Construction of a Transnational Legal Order (le premier écrit avec Bryant Garth ${ }^{8}$ ), creusait ce même sillon à partir cette fois d'une enquête globale sur l'arbitrage commercial international en construisant un cadre d'analyse des intersections entre élites juridiques nationales en concurrence et gouvernance globale : par une analyse multi-située avant l'heure qui multiple les vignettes empiriques locales (à Paris, Genève, Le Caire, etc.) et les analyses biographiques, il montre comment le champ global de l'arbitrage est structuré par les concurrences entre les avocats professionnels produits en masse par les law schools américaines aux prises avec « le petit club des professeurs et des avocats seniors européens cosmopolites ayant des connaissances généralistes », nouant ainsi dans une même trame les espaces de pouvoir et les élites nationales et transationales.

Pourtant, c'est certainement avec La mondialisation des guerres de palais. La restructuration du pouvoir d'État en Amérique Latine. Entre notables du droit et "Chicago Boys 9 " que cette sociologie de l'internationalisation du droit et des élites juridiques est devenue plus largement une analyse structurale de la globalisation et des luttes de pouvoir comme des rapports de domination qui l'organisent. Rompant radicalement avec le saucissonage des ana-

7. Bourdieu P., «Les conditions sociales de la circulation internationale des idées », Actes de la recherche en sciences sociales, $\mathrm{n}^{\circ} 145,2002$, pp. 3-8.

8. Dezalay Y., Garth B., Dealing in Virtue. International Commercial Arbitration and the Construction of a Transnational Legal Order, Chicago, University of Chicago Press, 1998.

9. Dezalay Y., Garth B., La mondialisation des guerres de palais. La restructuration du pouvoir d'État en Amérique Latine. Entre notables du droit et "Chicago Boys ", Paris, Seuil, 2002. 
lyses multi-niveaux alors en vogue, l'enquête articule les batailles professionnelles et politiques des « centres » (surtout américains) et des " périphéries » latino-américaines (Argentine, Brésil, Chili et Mexique). Il fait ainsi apparaître la zone grise qui se développe à l'intersection des champs de pouvoir nationaux du Nord et du Sud, et qui s'analyse comme un champ de l'expertise étatique transnationale en pleine expansion où se croisent universitaires de l'Ivy League, militants d'ONG, entreprises multinationales, fondations philanthropiques, institutions financières internationales, cabinets d'avocats américains. Plutôt qu'une vision unidimensionnelle de la domination (top-down) telle que proposée par les travaux néo-gramsciens centrés sur la formation d'une classe capitaliste transnationale ${ }^{10}$, Bryant Garth et Yves Dezalay prennent au sérieux cette couche transnationale épaisse d'import-export des savoirs d'État qu'ils saisissent dans ses différents pôles complémentaires et concurrents - du marché et de la vertu, de l'économie et du droit, du Nord et du Sud. Loin d'être un simple espace de diffusion ou de circulation des idées, ce champ transnational de l'expertise réformatrice d'État est un vaste espace de mise en concurrence des capitaux familiaux, experts, politiques (nationaux et internationaux), où se définissent et se redéfinissent les cadres sociaux et professionnels de la domination à l'échelle nationale et globale. Ils offraient ainsi une voie de dépassement des travaux dits « transnationalistes » marqués par un prisme diffusionniste et circulatoire mal ajusté à l'analyse des formes de domination ${ }^{11}$, mais aussi des théories post-marxistes de la dépendance centrées sur la seule domination économique : pour eux, les processus hégémoniques s'inscrivent dans des circuits de domination symbolique concurrents (des Chicago boys et des ONG des droits de l'homme) et ils s'analysent comme un effet de champ par lequel des périphéries telles que le Chili ou le Brésil se sont trouvées prises et enrôlées dans le champ gravitationnel des batailles professionnelles et expertes (« guerres de palais ») se déroulant dans le champ de pouvoir américain.

Cette boussole à la main, Bryant Garth et Yves Dezalay sont devenus des sociologues globe-trotter engageant un aller-retour continu entre l'Amérique du Nord d'une part, et l'Amérique latine mais aussi l'Asie qui est au cœur de leurs deux derniers ouvrages, d'autre part ${ }^{12}$. Il pourra sembler surprenant que l'Europe n'ait jamais, ou presque, été un objet de leurs recherches, mais c'est qu'elle ne cesse en fait d'apparaitre au miroir des terrains périphériques - validant ici encore l'intérêt heuristique d'une stratégie de recherche qui consiste à s'éloigner des puissances hégémoniques pour mieux en saisir l'écho. Y com-

10. Voir l'article de François-Xavier Dudouet et Antoine Vion dans ce numéro.

11. En ce sens, Vauchez A., "Le prisme circulatoire. Retour sur un leitmotiv académique ", Critique internationale, $\mathrm{n}^{\circ} 2,2013$, pp. 9-16.

12. Garth B., Dezalay Y., Asian Legal Revivals Lawyers in the Shadow of Empire, Chicago, University of Chicago Press, 2010 ; Garth B., Dezalay Y., Law as Reproduction and Revolution. An Interconnected History of the Internationalization of National Legal Hierarchies, Berkeley, University of California Press, 2021, à paraitre. 
pris quand il s'agit d'aller chercher dans l'Europe des premières facultés de droit des XII et XIII e siècles, les clés d'analyse d'une sociologie comparée des champs professionnels du droit et de leur encastrement social, économique et politique. Si de cette genèse commune naîtra un large éventail de variantes nationales (hiérarchie changeante des professionnels du droit, proximité inégale au monde des affaires comme au champ politico-administratif, etc.), Bryant Garth et Yves Dezalay identifient depuis leurs terrains asiatiques les caractéristiques historiquement stables de cette matrice européenne des champs du pouvoir juridique : cette formule singulière du capital juridique comme combinatoire changeante de capitaux sociaux hérités et de capitaux méritocratiques, de logiques de reproduction familiale et de méritocratie scolaire, sans lesquels la pérennité du droit et de ses élites par-delà les révolutions politiques et religieuses serait incompréhensible ; mais aussi cette position carrefour du droit dans le champ du pouvoir « où le capital social est converti plus facilement en diverses autres formes de capital : économique, intellectuel, politique ${ }^{13}$ ». Mais l'Europe réapparaît aussi par le prisme des sociétés impériales puisque c'est bien la concurrence entre les empires européens qui aura ouvert la voie à la formation d'un champ transnational de technologies de «bonne gouvernance ». Les périphéries (post-)coloniales n'étaient pas seulement un champ de bataille entre empires mais bien aussi, au sein même des "sociétés impériales », un espace de concurrence entre élites, groupes professionnels et formes d'expertise (en particulier le droit, l'économie) en concurrence pour ré-inventer le gouvernement colonial mais aussi, après l'indépendance, les relations avec l'ancienne métropole. Les empires européens auront participé à faire exister un premier marché d'import-export de la connaissance étatique. Reste à savoir si, figurant ainsi en point de départ et en matrice, l'Europe figure aussi en point d'arrivée, et dans quelle mesure elle est à son tour devenue une périphérie à part entière où s'observent à l'Est comme à l'Ouest du continent les traces des nouvelles batailles hégémoniques, américaines bien sûr mais aussi aujourd'hui chinoises.

\section{Tournant sociologique des relations internationales et tournant interna- tional de la sociologie politique}

Ce qui fait en effet l'un des intérêts majeurs des travaux d'Yves Dezalay, c'est que, sans pourtant avoir jamais cherché à faire école, il se situe au croisement de deux évolutions essentielles des sciences sociales de l'international au cours des deux dernières décennies : à la fois un "tournant " sociologique 14 des relations internationales centré sur les pratiques 15 mais aussi un « tour-

13. Charle C., "Pour une histoire sociale des professions juridiques », Actes de la recherche en sciences sociales, vol. 76-77, 1989, pp. 117-119.

14. Brast B., “A Sociological Turn in Statebuilding Research?”, Journal of Intervention and Statebuilding, $\mathrm{n}^{\circ} 4,2013$, pp. 530-540.

15. Olsson C., "Interventionism as Practice: On 'Ordinary Transgressions' and their Routinization”, Journal of Intervention and Statebuilding, n4, 2015, pp. 425-441 ; Pomarede 
nant » international de la sociologie politique ${ }^{16}$. Sur ce dernier point, l'année de la publication de La mondialisation des guerres de Palais (2002) apparaît rétrospectivement comme une date charnière pour une nouvelle génération d'internationalistes. De manière frappante, elle marque en France à la fois l'une des rares incursions de Pierre Bourdieu dans l'espace international à propos de la circulation des idées ${ }^{17}$, mais aussi un premier déplacement de la sociologie politique vers les terrains transnationaux en suivant les conditions des engagements du travail humanitaire 18 comme des mouvements sociaux liés à l'altermondialisme ${ }^{19}$. Si les styles, les positionnements et les objets diffèrent, il y a bien une assise épistémologique fermement partagée : une analyse incarnée des formes d'engagement et de mobilisation à l'international, une approche par les champs attentive aux normes et aux temporalités spécifiques, une méthode prosopographique longitudinale inscrite dans des configurations historiques, un souci permanent pour équilibrer effets de structures et trajectoires individuelles dans l'analyse des rapports de domination, une vigilance à la diversité des arènes à travers lesquelles circule l'expertise. Peu importe ici de savoir si la sociologie politique de l'international constitue une alternative au sein d'une sous-discipline " américano-centrée 20 », une « French connexion 21 » ou, au contraire, un nationalisme méthodologique archaïque favorisant la ghettoïsation des internationalistes français ${ }^{22}$, car l'enjeu est plutôt de resituer les travaux de Dezalay dans cette généalogie.

Son positionnement ouvre donc la voie à l'importante entreprise de «banalisation » des relations internationales au cours de la décennie 2000 qui remet en question tout ce qui pourrait s'apparenter à un découplage ou à des formes d'extraterritorialité ontologique ou épistémologique des phénomènes internationaux par rapport aux faits sociaux. En ce sens, la portée et l'héritage de ses travaux sur la génération d'internationalistes entreprenant une thèse

J., « Aux prises avec les vibrations du social : retour sur le Practice turn en Relations Internationales ", Cultures $\&$ Conflits, ${ }^{\circ}{ }^{102}$, pp. 151-164.

16. Siméant-Germanos J., Guide de l'Enquête Globale en Sciences Sociales, Paris, CNRS Éditions, 2015. En ce sens, voir aussi le panel : «Que fait l'étude des relations internationales à la sociologie politique ?» du Réseau Thématique 34 du Congrès 2019 de l'Association Française de Sociologie (AFS), https://sociopo.hypotheses.org/740, consulté le 7 juin 2019.

17. Bourdieu P., "Les conditions sociales de la circulation internationale des idées ", Actes de la recherche en sciences sociales, $\mathrm{n}^{\circ} 145,2002$, pp. 3-8.

18. Dauvin P., Siméant J., Le travail humanitaire. Les acteurs des ONG, du siège au terrain, Paris, Presses de Science Po, 2002.

19. Agrikoliansky E., La Ligue française des droits de l'homme et du citoyen depuis 1945. Sociologie d'un engagement civique, Paris, L'Harmattan, 2002.

20. Basaran T., Bigo D., Guittet E.-P., Walker R. B. J., International Political Sociology: Transversal Lines, Londres, Routledge, 2016.

21. Guilhot N., " "The French Connection". Éléments pour une histoire des relations internationales en France », Revue française de science politique, vol. 67, n 1, 2017, pp. 43-67.

22. Cornut J., Battistella D., "Is French IR Emerging? French IR Scholars in the 2011 TRIPP Survey”, Revue Française de Science Politique, vol. 63, n², 2013, pp. 303-336 ; Balzacq T., Cornut J., Ramel F., "Global International Relations as Alternative to the American Mainstream: The Case of International Relations in France", Critique Internationale, ${ }^{\circ} 1$, 2017, pp. 69-93. 
durant cette période est tout à fait considérable. Sans jamais explicitement se situer dans ce type de débats, ces travaux ont contribué à ramener l'étude des phénomènes internationaux dans le giron des sciences sociales en y puisant ses outils « ordinaires » d'investigation ${ }^{23}$. De fait, La mondialisation des guerres de palais, avec notamment les travaux de Didier Bigo sur la sécurité ${ }^{24}$ ou d'Anne-Catherine Wagner sur l'immigration ${ }^{25}$, précède la fortune contemporaine des concepts bourdieusiens en relations internationales (champs, capitaux, habitus) pour expliquer les rapports de domination à l'international 26 ou l'internationalisation des groupes professionnels comme les spécialistes de la paix ${ }^{27}$, les " gestionnaires » de crises internationales 28 ou les juristes et bureaucrates dans le champ du pouvoir européen ${ }^{29}$. Ce sont donc ces passerelles multiples avec la sociologie politique (reproduction des noblesses d'État, import-export des savoirs, etc.) et avec les relations internationales que ce numéro ambitionne de discuter.

Mais ce dossier doit également permettre de pointer certaines interrogations encore pendantes. L'examen critique fait partie intégrante de l'héritage que constitue la sociologie d'Yves Dezalay. On pense ici à l'hypothèse centrale mais inégalement heuristique d'une homologie structurale pour expliquer la convergence des modèles de gouvernement centre/périphérie et le succès des stratégies d'import/export : si l'homologie est idéal-typique entre l'université catholique de Santiago et l'école de Chicago, les autres comparaisons Nord/Sud se réfèrent davantage à une «zone floue » de contact entre «dominés des dominants et dominants des dominés ». Au plan méthodologique, si l'identification des arènes et l'approche comparative entre périphé-

23. Bigo D., Walker R. B. J, "International, Political, Sociology", International Political Sociology, $\mathrm{n}^{\circ} 1,2007$, pp. 1-5 ; Siméant-Germanos J., Guide de l'enquête globale, op. cit.

24. Bigo D., "Grands débats dans un petit monde », Cultures E Conflits, n¹9-20, 1995, pp. 648.

25. Wagner A.-C., Les nowvelles élites de la mondialisation. Une immigration dorée en France, Paris, Presses Universitaires de France, 1998.

26. Mérand F., Pouliot V., « Le Monde de Pierre Bourdieu: Éléments pour une Théorie Sociale des Relations Internationales », Canadian Journal of Political Science, n³, 2008, pp. 603-625; Adler-Nissen R., Bourdieu in International Relations: Rethinking Key Concepts in IR, Abingdon, Routledge, 2012.

27. Autesserre S., Peaceland: Conflict Resolution and the Everyday Politics of Intervention, New York, Cambridge University Press, 2015 ; Bliesemann De Guevara B. (ed.), Statebuilding and State-Formation: The Political Sociology of Intervention, Londres Routledge, 2012 ; Goetze C., The Distinction of Peace. A Social Analysis of Peacebuilding, Ann Arbour, University of Michigan Press, 2017 ; Daho G., Duclos N., Jouhanneau C., "Political Sociology of International Interventions. Peacebuilders and the Ground", Journal of Intervention and Statebuilding, $\mathrm{n}^{\circ}$ 3, 2019, pp. 1-14.

28. Ambrosetti D., Buchet de Neuilly Y., «Les organisations internationales au cœur des crises », Cultures E Conflits, n75, 2009, pp. 7-14 ; Daho G., La transformation des armées. Enquête sur l'institutionnalisation des relations civilo-militaires en France, Paris, Éditions de la Maison des Sciences de l'Homme, 2016, notamment le chapitre 9.

29. On renvoie ici plus particulièrement aux auteurs réunis dans le dossier « Constructions européennes " d'Actes de la recherche en sciences sociales ( ${ }^{\circ} 166-167,2007$, coordonné par Antonin Cohen, Yves Dezalay et Dominique Marchetti); et Georgakakis D., Vauchez A., "Le champ à l'épreuve de l'Europe ", in Siméant J. (dir.), Guide de l'enquête globale, op. cit. 
ries sont relativement stabilisées, les matériaux empiriques reposent essentiellement sur la conduite d'entretiens à une échelle que nous pourrions aisément qualifier d'industrielle (près de 4000 entretiens cumulés dans une quarantaine de pays) même si l'auteur se plait à souligner la dimension bricolée de son travail empirique. L'exploitation et la mise en valeur souvent allusive de ces entretiens, sous forme de vignettes illustratives en fin de chapitre, ne rend pas forcément justice à la sophistication du travail prosopographique, ou, pour le dire avec l'auteur, de "biographie du champ ». Le système d'administration de la preuve y est d'une telle densité qu'il peut rendre la lecture de l'ouvrage abrupte d'autant qu'Yves Dezalay se montre méfiant à l'égard des techniques d'objectivation statistique. La difficulté de transmission de la sociologie d'Yves Dezalay vis-à-vis des étudiants, qui peut expliquer le fait que la portée de ses travaux demeure encore trop peu discutée, repose sur un grand écart entre l'exigence et le coût d'entrée empirique des ouvrages d'un côté, et de l'autre, le caractère plus panoramique de certains articles, souvent taillés à la serpe ${ }^{30}$. Le politiste évoquera aussi un angle mort qui gagne à être discuté : celui des dynamiques institutionnelles (inégalement) autonomes. Même si les arènes de l'import-export des savoirs d'État sont clairement identifiées (Ivy League, Foreign Policy Establishment, institutions financières internationales, fondations philanthropiques, etc.), les travaux d'Yves Dezalay nous en disent peu sur les «structures institutionnelles » et les sentiers de dépendance du système international, c'est-à-dire de ce qui « fonctionne » et « tient » de façon relativement indépendante de l'intentionnalité et de l'agenda des agents-doubles.

\section{Singularité, mise à l'épreuve et discussion des recherches d'Yves Dezalay}

Ce dossier vise donc à enrichir cette discussion. Il est articulé en trois moments distincts : une réflexion transversale qui resitue l'originalité des travaux d'Yves Dezalay au cœur de l'analyse des rapports de domination internationaux, une mise à l'épreuve empirique de ses concepts clés autour du déploiement des expertises internationales dans le domaine juridique et une discussion collégiale sur ses apports en matière de stratégies d'ouverture de terrain.

Ce numéro propose une réflexion transversale qui cherche à identifier et à mettre en relation la sociologie de l'international d'Yves Dezalay dans l'espace des sciences sociales de l'international. Il s'agit tout d'abord de resituer à la fois la singularité théorique, épistémologique et méthodologique de son approche sociologique de la mondialisation et ses usages au cœur d'une sociologie politique de l'international désormais très dynamique. Ainsi, l'article de François-Xavier Dudouet et d'Antoine Vion pointe la rupture avec l'application du marxisme dans les travaux d'Yves Dezalay dans la mesure où les élites n’y sont pas homogènes, les relations centre/périphérie pas univoques et la 
dépendance pas seulement économique ${ }^{31}$. Il souligne également l'originalité d'une entrée dans les rapports de domination par le biais des savoirs d'État (droit et économie) mais aussi de l'exportation des «hiérarchies nationales » du centre vers les périphéries.

Le dossier propose ensuite une mise à l'épreuve empirique de certains de ses concepts-clés. Â partir d'un terrain déjà exploité sur les « pacificateurs » et autres professionnels de la justice transitionnelle, Delphine Griveaud et Sandrine Lefranc questionnent une contradiction interne aux travaux d'Yves Dezalay qui caractérise les espaces internationaux comme "zone floue et fluctuante » tout en leur appliquant la loi d'airain de l'homologie structurale. De son côté, s'appuyant sur une enquête en cours sur les avocats (notamment parisiens) impliqués dans la négociation de contrats miniers et pétroliers africains, Sara Dezalay revisite la notion «d'espace carrefour » et pointe son caractère heuristique pour tracer les reconfigurations continues des stratégies politiques, économiques, morales et sociales des membres du «barreau africain ». Enfin Mikael Madsen teste la validité de la thèse de la domination du champ (européen et international) des droits de l'homme par une élite de « juristes-politiciens du droit » au prisme de la politisation de la question des droits de l'homme et de la contestation multiforme dont fait aujourd'hui l'objet la Cour européenne des droits de l'homme.

Le dossier se conclut par une discussion sur l'enquête "à l'international » autour d'une table-ronde réunissant un panel diversifié d'auteurs qui ont croisé les travaux d'Yves Dezalay au fil de divers terrains et échanges intellectuels : Afrânio Garcia (EHESS, CESSP), Didier Bigo (Kings College, Sciences Po Paris), Laurent Jeanpierre (Paris 1, CESSP), Ron Levi (Université de Toronto) et Johanna Siméant (ENS, CMH). Repartant des usages et des conditions variées de réception des travaux d'Yves Dezalay, le panel propose une réflexion à plusieurs voix sur les stratégies d'enquête à l'international comme les techniques spécifiques d'ouverture de terrains, et ouvre à un débat plus large sur « le tournant global des sciences sociales».

Ce numéro constitue également l'occasion d'accueillir un article en horsthème consacré à la violence stratégique et l'auto-défense en Pays Basque. Au moyen d'une enquête menée par entretiens auprès d'une soixantaine de militants clandestins de l'Euskadi Ta Askatasuna (ETA), Caroline Guibet Lafaye revient sur les représentations de la violence. Elle substitue au niveau méso traditionnel, interprétant la violence sous le prisme stratégique du choix rationnel, le niveau microsocial attentif au paradigme de l'auto-défense.

30. Dezalay Y., « Les courtiers de l'international. Héritiers cosmopolites, mercenaires de l'impérialisme et missionnaires de l'universel ", Actes de la recheche en sciences sociales, n¹51-152, 2004, pp. 4-35.

31. Amin S., Le développement inégal. Essai sur les fomations sociales du capitalisme périphérique, Paris, Éd. de Minuit, 1973. 
La chronique bibliographique, rédigée par Christophe Wasinski, propose la recension et la discussion de trois ouvrages portant sur l'usage des armes non létales par les forces de sécurité : Anna Feigenbaum (Petite histoire du gaz lacrymogène. Des tranchées de 1914 aux gilets jaunes, Paris, Libertalia, 2019), Paul Rocher (Gazer, mutiler, soumettre. Politique de l'arme non létale, Paris, La Fabrique, 2020) et Juliette Volcler (Le son comme arme. Les usages policiers et militaires du son, Paris, La Découverte, 2011).

Le numéro propose enfin un entretien conduit par Hager Ben Jafel avec Antoine Vauchez autour de l'expérience de l'écriture et de la diffusion d'une proposition de traité de démocratisation de l'Union européenne (2017-2019) qui est l'occasion d'une réflexion sur les conditions et les formats d'intervention des chercheurs en sciences sociales dans le débat public français et européen. 